\title{
Type-2 kernelized fuzzy c-means algorithm based on the uncertain width of Gaussian kernel with applications in MR image segmentation
}

\author{
Qinli Zhang ${ }^{1, a^{*}}$, Yajun $\mathrm{Bi}^{1, \mathrm{~b}}$ and Zhigang Gong ${ }^{3, \mathrm{c}}$ \\ ${ }^{1}$ Dept. of Fundamental Science, North China Institute of Aerospace Engineering, Langfang China \\ ${ }^{2}$ The factory attached to institute, North China Institute of Aerospace Engineering, Langfang China \\ aZZhangql1973@163.com, bstl1972@163.com, ccp_dd@sina.com
}

Keywords: KFCM; Type-2 fuzzy sets; Gaussian kernel; MR image.

Abstract. While fuzzy c-means is a popular soft clustering method, its effectiveness is largely limited to spherical clusters. By applying kernel tricks, the kernel fuzzy c-means algorithm attempts to address this problem by mapping data with nonlinear relationships to appropriate feature spaces. Kernel width is crucial for effective kernel clustering. Unfortunately, for most applications, it is not easy to find the right width. To design and manage the uncertainty for kernel width, we propose a type- 2 kernelized fuzzy c-means algorithm (T2KFCM). We extend the type-1 fuzzy sets of membership to interval type-2 fuzzy sets using two widths and which creates a footprint of uncertainty for the membership. Experiments on MR (Magnetic Resonance) image are given that compare kernelized FCM (KFCM) with T2KFCM. The results show that T2KFCM compares favorably to both of the previous models.

\section{Introduction}

MR image plays a vital role in medical environments. The biggest challenge in MR image segmentation is the spatial intensity inhomogeneity induced by the radio-frequency coil[1]. Although the improving progress of fuzzy techniques, e.g. KFCM has relieved this difficulty to a certain extent, the challenge still exists and becomes the kernel width selection [2-4]. The performance of KFCM significantly depends on the choice of . Hence, a good choice of is crucial. Unfrotunately, it is unclear which kernels are more suitable for a particular task, because is heavily influenced by prior knowledge about the data and patterns that we expect to discover [5]. As a result of uncertain, these KFCMs can not respond identically to different patients. They can not directly model all kinds of uncertainties in MR image segmentation. They usually produce over segmentation and also give unsatisfied results.

Therefore, to address the above problems , a novel MR image segmentation algorithm is proposed by bringing type- 2 fuzzy sets into KFCM. Some researchers have shown that the uncertainty in fuzzy systems can be captured with type-2 fuzzy sets because of their three-dimensional membership function. Type- 2 membership functions are fuzzy and can appropriately model all kinds of uncertainties in real application problems. When clustering methods are combined with type- 2 fuzzy sets, the prototype data can be clustered more properly and accurately[6-7]. We extend the type-1 membership values to interval type-2 membership values using two kernel widths and which create a footprint of uncertainty (FOU) of membership values. Then the interval T2KFCM is proposed by incorporating this interval type-2 fuzzy sets into KFCM. The experimental results are given to show the superiority of T2KFCM in comparison with KFCM.

The rest of this paper is organized as follow. Section II describes the KFCM with spatial information. Section III describes the interval T2KFCM with spatial information, Section IV provides an example showing the validity of our proposed medhod, and finally Section V concludes the work.

\section{KFCM with spatial informations}

There is a trend in machine learning community to construct a nonlinear version of a linear algorithm using the kernel method, e.g., Support Vector Machines (SVM), Kernel Fisher Discriminant (KFD) and Kernel Principal Component Analysis (KPCA) [8]. The philosophy of the 'kernel method' is that 
every linear algorithm that only uses scalar products can be extended to the corresponding nonlinear version of this algorithm which is implicitly executed in a higher feature space through kernels. In one of our early works, a novel kernel-based fuzzy c-means algorithm with spatial constraints is derived from this philosophy [9]. The algorithm adopts a new kernel-induced distance metric to replace the original squared-norm one in FCM and utilizes the spatial information that is important in image clustering. It is shown to be more robust to noise and outlier than classical algorithms[10]. The modified objective function is given as follows:

$$
J=\sum_{i=1}^{c} \sum_{k=1}^{N} u_{i k}^{m}\left(1-K\left(x_{k}, v_{i}\right)\right)+\frac{\alpha}{N_{R}} \sum_{i=1}^{c} \sum_{k=1}^{N} u_{i k}^{m} \sum_{r \in N_{k}}\left(1-K\left(x_{r}, v_{i}\right)\right)
$$

where $N_{k}$ stands for the set of neighbors that exist in a window around $x_{k}$ and $N_{R}$ is the cardinality of $N_{k}$. The parameter $\alpha$ controls the effect of the penalty term. The relative importance of the regularizing term is inversely proportional to the signal-to-noise (SNR) ratio of the image. In other words, Lower SNR would require a higher value of the parameter $\alpha$, and vice versa. $K(x, y)$ is an inner product kernel function. We adopt the Gaussian RBF kernel function, i.e. , $K(x, y)=\exp \left(-\|x-y\|^{2} / 2 \sigma^{2}\right)$, where $\sigma$ is the kernel width.

An iterative algorithm of minimizing Eqs. (1) can be derived by evaluating the centroids and membership functions that satisfy a zero gradient condition. A necessary condition for Eqs. (1) to be at a local minimum is

$$
u_{i k}=\frac{\left(\left(1-K\left(x_{k}, v_{i}\right)\right)+\frac{\alpha}{N_{R}} \sum_{r \in N_{k}}\left(1-K\left(x_{r}, v_{i}\right)\right)\right)^{\frac{-1}{m-1}}}{\sum_{j=1}^{c}\left(\left(1-K\left(x_{k}, v_{j}\right)\right)+\frac{\alpha}{N_{R}} \sum_{r \in N_{k}}\left(1-K\left(x_{r}, v_{j}\right)\right)\right)^{\frac{-1}{m-1}}} \quad, \quad v_{i}=\frac{\sum_{k=1}^{n} u_{i k}^{m}\left(K\left(x_{k}, v_{i}\right) x_{k}+\frac{\alpha}{N_{R}} \sum_{r \in N_{k}} K\left(x_{r}, v_{i}\right) x_{r}\right)}{\sum_{k=1}^{n} u_{i k}^{m}\left(K\left(x_{k}, v_{j}\right)+\frac{\alpha}{N_{R}} \sum_{r \in N_{k}} K\left(x_{r}, v_{j}\right)\right)} .
$$

\section{Interval type-2 KFCM with spatial informations}

With respect to the selection of the kernel function, to determine the parameters of the kernel function is more important.The kernelized fuzzy c-means with Gaussian RBF kernel is sensitive to the kernel width $\sigma$ that greatly affects clustering segmentation. Kernel width is crucial for effective kernel clustering. If the clusters in a pattern set are of different size and density, the performance of KFCM may significantly differ depending on the choice of the kernel width. Hence, a good choice of $\sigma$ may be considered to be related to the distribution of the patterns for the pattern set. If we set $\sigma$ to a large value, the distance between patterns is shortened and the pattern will tend to be very similar. This may seem to be desirable for the sparse data, however, the estimated cluster centers will tend to be closer to each other. If we set $\sigma$ to a mall value, the distance between patterns is widened and the pattern will tend to be very dissimilar. This may seem to be desirable for the dense data, however, the estimated cluster centers will tend to be more distant to each other.

The value of $\sigma$ should reflect the radical scope of the input data. Unfortunately, for most applications, it is uneasy or impossible to precisely specify the appropriate fixed value of $\sigma$ to be used in KFCM because the unevenness of data is out of the reach of the strength of constant kernel width. However, if we can somehow simultaneously incorporate various values of $\sigma$, we may perhaps be able to design a desirable KFCM. Therefore, we should consider memberships for a pattern as uncertain instead of as certain in the KFCM. In other words, memberships for a pattern should not have to rely on a single specific kernel width $\sigma$ as in KFCM. We compute an interval of primary memberships for a pattern with two kernel widths $\sigma_{1}$ and $\sigma_{2}$ which represent different radical scope. The establishment of the membership interval for a pattern set is to represent and manage the uncertainty which occurs by the use of $\sigma_{1}$ and $\sigma_{2}$ in KFCM. Hence, we extend a pattern set to interval type-2 fuzzy sets. Interval type-2 kernel-based fuzzy c-means algorithm with spatial constraints (T2KFCM) is proposed. 
T2KFCM is extension of KFCM by using two kernel parameters $\sigma_{1}$ and $\sigma_{2}$ to make footprint of uncertainty, corresponding to upper and lower membership values of KFCM. The use of kernel width gives different objective functions to be minimized as follows:

$$
\begin{aligned}
& J_{\sigma_{1}}=\sum_{i=1}^{c} \sum_{k=1}^{N} u_{i k}^{m}\left(1-K_{\sigma_{1}}\left(x_{k}, v_{i}\right)\right)+\frac{\alpha}{N_{R}} \sum_{i=1}^{c} \sum_{k=1}^{N} u_{i k}^{m} \sum_{r \in N_{k}}\left(1-K_{\sigma_{1}}\left(x_{r}, v_{i}\right)\right), \\
& J_{\sigma_{2}}=\sum_{i=1}^{c} \sum_{k=1}^{N} w_{i k}^{m}\left(1-K_{\sigma_{2}}\left(x_{k}, v_{i}\right)\right)+\frac{\alpha}{N_{R}} \sum_{i=1}^{c} \sum_{k=1}^{N} w_{i k}^{m} \sum_{r \in N_{k}}\left(1-K_{\sigma_{2}}\left(x_{r}, v_{i}\right)\right) .
\end{aligned}
$$

Upper and lower degrees of membership, $\bar{u}_{i j}$ and $\underline{u}_{i j}$ are determined as follows:

$\bar{u}_{i j}=\max \left(u_{i j}, w_{i j}\right), \quad \underline{u}_{i j}=\min \left(u_{i j}, w_{i j}\right)$.

Because each pattern has membership interval as the upper $\bar{u}$ and the lower $\underline{u}$, each centroid of cluster is represented by the interval between $v_{L}$ and $v_{R}$..

After obtaining $v_{i}^{L}$ and $v_{i}^{R}$, type-reduction is applied to get centroid of clusters as follows:

$v_{i}=\left(v_{i}^{L}+v_{i}^{R}\right) / 2$

The kernel width must adapt, to some extent, the space distribution. In dense areas the width is narrow, and in sparse areas the width is wide. Hence, we propose a method of computing the kernel widths $\sigma_{1}$ and $\sigma_{2}$ according to the distance between iuput vectors. Assume we have a data set $S$ with $l$ patterns $x_{j}(j=1, \mathrm{~L}, l)$ and is divided into $c$ clusters $v_{i}(i=1, \mathrm{~L}, c)$. Now, we describe how to compute $\sigma_{1}$ and $\sigma_{2}$.

Let $\sigma_{0}^{2}=\frac{1}{c} \sum_{i=1}^{c} \sigma_{i}^{2}$, where $\sigma_{i}^{2}=\frac{1}{k} \sum_{j=1}^{k}\left\|x_{j}-v_{i}\right\|^{2}, k$ is the number of the neighbors of the $i$ th center.

The factors $f_{v}$ controls the intervals in which the parameter varies: $\sigma_{1}=f_{v} \sigma_{0}, \sigma_{2}=\frac{1}{f_{v}} \sigma_{0}$.

Because the Gaussian function has $99.7 \%$ of its probability mass in the range of $[\mu-3 \sigma, \mu+3 \sigma]$, we constrain $f_{v} \in[1 / 6,1]$.

\section{Experimental demonstrations}

In this section, we describe some experimental results to compare the segmentation performance of the following algorithm, i.e. KFCM and T2KFCM.The experiment was perfomed on a real MR slice. In order to state our experimental results fairly, we run the T2KFCM algorithm with fifty iterations. All experiments were run on a computer with 2 GB memory, Microsoft Windows 8 and Matlab 2012b. Figs 1-3 show the original MR images and segmented results using KFCM and T2KFCM algorithm.

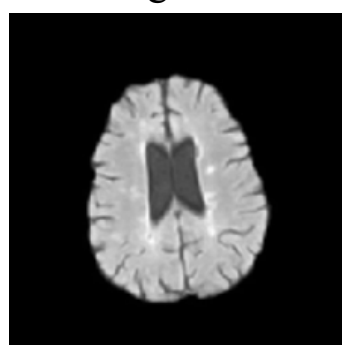

Fig. 1 Original image

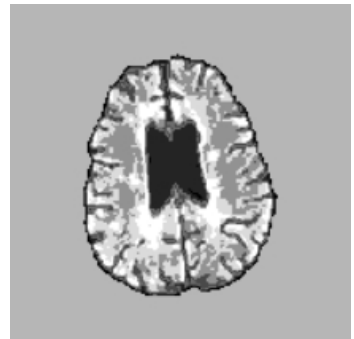

Fig. 2 KFCM result

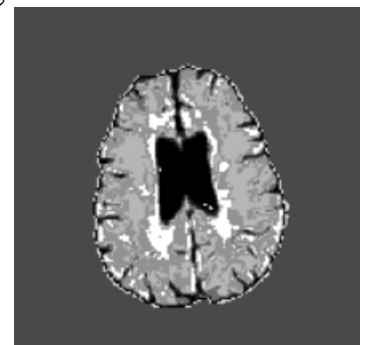

Fig. 3 T2KFCM result

From the above images, we can see that the proposed method gives good results and the regions are distinct. 


\section{Conclusions}

In this paper, we extended a type-1 fuzzy set to an interval type- 2 fuzzy set by incorporating two different values of kernel width $\sigma\left(\sigma_{1}\right.$ and $\left.\sigma_{2}\right)$ and modified the procedure of KFCM. The purpose was to represent and manage the uncertainty which occurred in irregular MR image. Consequently, the management of uncertainty aids the cluster centers to converge to a more reasonable position in our proposed method. Center-updating and hard partition was modified in the KFCM by incorporating interval type-2 fuzzy sets into KFCM. From the approach, we can obtain improved segmentation results because the prototype data can be clustered more properly and accurately. An example for MR image illustrated the effectiveness of our proposed method by comparison with FCM and KFCM.

\section{Acknowledgement}

I would like to thank all my friends who have helped me develop the fundamental and essential academic competence. This paper is supported by doctoral fund of NCIAE (No. BKY-2014-09).

\section{References}

[1] D.-Q. Zhang and S.-C. Chen, Clustering incomplete data using kernel-based fuzzy c-means algorithm, Neural Process Letters, 18( 2003)155-162.

[2] Bing Nan Li , Chee Kong Cui, Stephen Chang and S.H. Ong., Integrating spatial fuzzy clustering with level set methods for automated medical image segmentation, Computers in Biologly and Medicine, 41(2011)1-10.

[3] H. Shen,J. Yang,S. Wang and X. Liu, Attribute weighted mercer kernel-based fuzzy clustering algorithm for general non-spherical datasets, Soft Computing, 10( 2006)1061-1073.

[4] Hsin-Chien Huang, Yung-Yu Chuang and Chu-Song Chen, Multiple Kernel Fuzzy clustering, IEEE Transactions on Fuzzy Systems, 20(2012)120-134.

[5] Zhang Chen, Xia Shixiong and Liu Bing, A Robust fuzzy Kernel clustering algorithm, Applied Mathematics and Information Sciences, 7( 2013)1005-1012.

[6] D. Graves and W. Pedrycz, Kernel-based on fuzzy clusteing and fuzzy clustering: A comparative experimental study, Fuzzy Sets System, 161(2010)522-543.

[7] Cheul Hwang and Frank Chung-Hoon Rhee, Uncertain fuzzy clustering: Interval type-2 fuzzy approach to C-means, IEEE Transactions on Fuzzy Systems, 15(2007)107-120.

[8] Muller K. R., Mika S., et al., An introduction to kernel-based learning algorithm, IEEE Transactions on Neural Networks, 12(2001)181-202.

[9] Daoqiang. Zhang and Songcan. Chen, A novel kernelized fuzzy c-means algoritm with applications in medical image segmentation, Artificial Intelligence in Medicine, 32(2004)7-50.

[10] Songcan Chen and Daoqiang Zhang, Robust Image Segmentation Using FCM With Spatial Constraints Based on New Kernel-Induced Distance Measure, IEEE Transactions on systems, man, and cybernetics_-part B: cybernetics, 34( 2007)1907-1916. 